\title{
Small-angle options of the upgraded ultrasmall-angle x-ray scattering beamline BW4 at HASYLAB
}

\author{
S. V. Roth, R. Döhrmann, M. Dommach, M. Kuhlmann, I. Kröger, and R. Gehrke \\ HASYLAB, DESY, Notkestrasse 85, D-22603 Hamburg, Germany \\ H. Walter \\ CSEM SA, Badenerstrasse 569, CH-8048 Zürich, Switzerland \\ C. Schroer \\ Institut für Strukturphysik, TU Dresden, D-01062 Dresden, Germany \\ B. Lengeler \\ II. Physikalisches Institut B, RWTH Aachen, D-52056 Aachen, Germany \\ P. Müller-Buschbaum \\ Physik-Department E13, Technische Universität München, James-Franck-Strasse 1, \\ D-85748 Garching, Germany
}

(Received 7 June 2006; accepted 17 July 2006; published online 18 August 2006)

\begin{abstract}
We present the upgrade and present status of the ultrasmall-angle x-ray scattering (USAXS) beamline BW4 at the Hamburg Synchrotronstrahlungslabor. In order to extend the accessible scattering vector range, new small-angle setups have been established, making use of the high flux and small divergence of BW4. In standard transmission geometry using a beam size of $B=400$ $\times 400 \mu \mathrm{m}^{2}$ (horizontal $\times$ vertical), typical small-angle resolution ranges from $d_{\max }=90$ to $650 \mathrm{~nm}$, depending on sample-to-detector distance. Additionally a new microfocus option has been established. This microfocus option allows reducing the sample size by one order of magnitude. Using parabolic beryllium compound refractive lenses, a new standard beam size of $B=65$ $\times 35 \mu \mathrm{m}^{2}$ (horizontal $\times$ vertical) can be provided. The $\mu$-SAXS resolution is as high as $d_{\max }$ $=150 \mathrm{~nm}$. Using $\mu$-SAXS in combination with grazing incidence ( $\mu$-GISAXS) on a standard noble metal gradient multilayer, we prove the feasibility of $\mu$-GISAXS experiments at a second generation source. (C) 2006 American Institute of Physics. [DOI: 10.1063/1.2336195]
\end{abstract}

\section{INTRODUCTION}

Small-angle $\mathrm{x}$-ray scattering (SAXS) is a powerful tool for material science. ${ }^{1-4}$ SAXS makes use of the penetration of $\mathrm{x}$ rays in materials, either in solid or liquid state. ${ }^{5}$ This feature renders SAXS an invasive but nondestructive method. X-ray scattering is caused by spatial electron density fluctuations. While wide-angle $\mathrm{x}$-ray scattering (WAXS) probes electron density fluctuations on the length scales of interatomic distances, typically scattering angles $2 \theta<5^{\circ}$ are considered as the SAXS regime. ${ }^{6}$ For these scattering angles $\mathrm{X}$-rays probe long-range fluctuations of the mean electron density over many atoms, thereby giving access to correlation length scales ranging from a few nanometers to several micrometers. The applications include a wide range of materials, e.g., nanoporous materials, polymers, colloids, and metals in bulk as well as in thin film geometry. ${ }^{4,6-9}$ Due to the high flux at second or third generation synchrotron radiation sources using wigglers or undulators, time-resolved experiments are standard. ${ }^{8,10-17}$ Routinely, heating or mechanical deformation is used to reveal structure-property relations in bulk material using transmission SAXS (TSAXS). SAXS combined with a reflection setup can be used to investigate thin films and surfaces using grazing incidence small-angle $\mathrm{x}$-ray scattering (GISAXS). ${ }^{8,18-23}$ Here, the incident $\mathrm{x}$-ray beam impinges under a very shallow angle (incident angle $\alpha_{i}<1^{\circ}$ ) onto the sample surface. Exploiting the finite penetration depth of totally reflected $\mathrm{x}$ rays into the material, this geometry is extremely surface sensitive and allows to investigate thin films down to nanometer in thickness. ${ }^{24,25} \mathrm{~A}$ key parameter characterizing SAXS measurements is the resolution in terms of the minimum accessible scattering angle $\theta_{\text {min }}$ which via Bragg's equation can also be expressed as the maximum correlation distance accessible in real space: $d_{\max }=\lambda /\left[2 \sin \left(\theta_{\min }\right)\right] \approx \lambda /\left(2 \theta_{\min }\right)$, with $\lambda$ denoting the wavelength used. ${ }^{26,27}$ Typically, a beam stop is used in TSAXS to block the incident direct beam. At a given sample-to-detector distance, $d_{\max }$ is determined by the size of this beam stop, which depends on the degree of collimation of the incident primary beam. ${ }^{26,27}$

The beamline BW4 at the DORIS III storage ring at HASYLAB has been designed as an ultrasmall-angle $\mathrm{x}$-ray scattering (USAXS) instrument with minimum scattering angles on the order of $0.01^{\circ}$. Due to its high flux and excellent collimation, BW4 achieves a maximum USAXS resolution of $d_{\max }>1 \mu \mathrm{m}$, as demonstrated in Fig. 1 for the USAXS pattern of active carbon. The original design of BW4 was described in Refs. 28-30. The x rays are produced by a wiggler ( $N=19$ periods, $K=13.2)$. The $\mathrm{x}$-ray beam is monochromatized using a fixed exit double $\mathrm{Si}(111)$ monochro- 


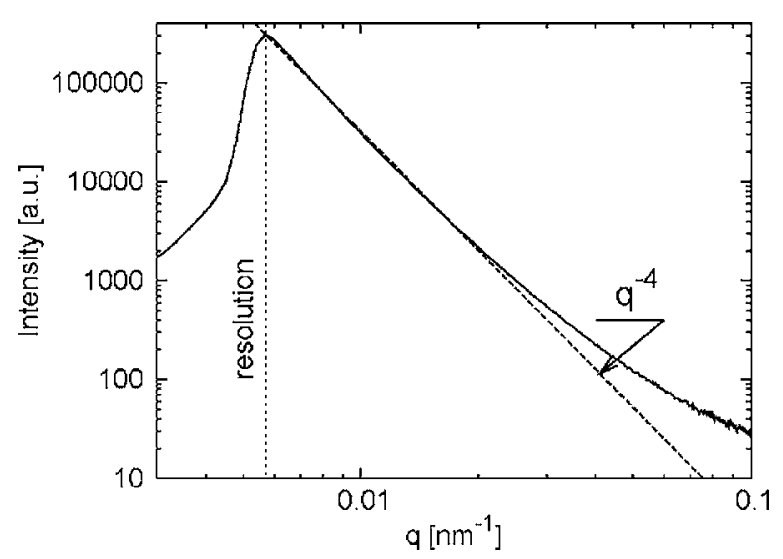

FIG. 1. USAXS pattern of active carbon. The solid line indicates a Porod fit. The deviation for large $q$ stems from the nanoporosity. The resolution at $L_{\mathrm{SD}}=12.4 \mathrm{~m}$ is $d_{\max }>1 \mu \mathrm{m}$. The beam-stop is off centered.

mator and focused horizontally and vertically using a fixed cylindrical mirror and a plane mirror with a mirror bender, respectively. ${ }^{31}$ For optimum resolution (minimum beam stop $\operatorname{size}^{26}$ ), the $\mathrm{x}$-ray beam is focused onto the detector. The distance between the collimating slits is about $L_{\text {coll }}=10 \mathrm{~m}$. In order to increase the accessible $q$ range, the beamline was significantly upgraded since 2003. The aim of this upgrade was to optimize flux and resolution for various shorter sample-to-detector distances below $8 \mathrm{~m}$ to increase the accessible $q$ range. The new options at BW4 include dedicated SAXS setups with sample-to-detector distances $L_{\mathrm{SD}}=1.8$, 2.3 , and $4 \mathrm{~m}$ with a fixed detector position determined by the fixed focal plane of the standard mirror geometry (horizontally focusing cylindrical and vertical focusing bent mirror). Furthermore, an option with a moderate microfocus ( $\mu$-focus) has become available in which the beam size is reduced to $B=65 \times 35 \mu \mathrm{m}^{2}$ or $B=30 \times 17 \mu \mathrm{m}^{2}$ (horizontal $\times$ vertical, $H \times V$ ), respectively. In the present article we describe the status of the upgraded instrument at BW4 and give an overview over the various SAXS options available now.

\section{SAXS POSITION}

A major aim of the BW4 upgrade was to get access to a couple of SAXS setups with different achievable resolution limits. The USAXS capabilities of the instrument had to be kept and the time for changing between the different setups had to be as short as possible.

Defined by the original USAXS setup the X-ray beam is focused to the fixed detection plane. This geometry guarantees a well defined incident beam with an optimum resolution and leads to high stability. ${ }^{26}$ Thus the detector position is kept fixed, and therefore the position of the sample environment has to be changed between the different SAXS and USAXS setups. To optimize intensity and resolution for the shorter sample-to-detector distances, the collimating system had to be changed and adapted. In order to avoid moving the guard slit $S_{2}$ of the USAXS setup which is permanently installed at a fixed distance of $42 \mathrm{~m}$ from the wiggler, ${ }^{31}$ an additional portable slit system was developed and implemented which allows to realize the required close distance between guard slit and sample for each SAXS setup.

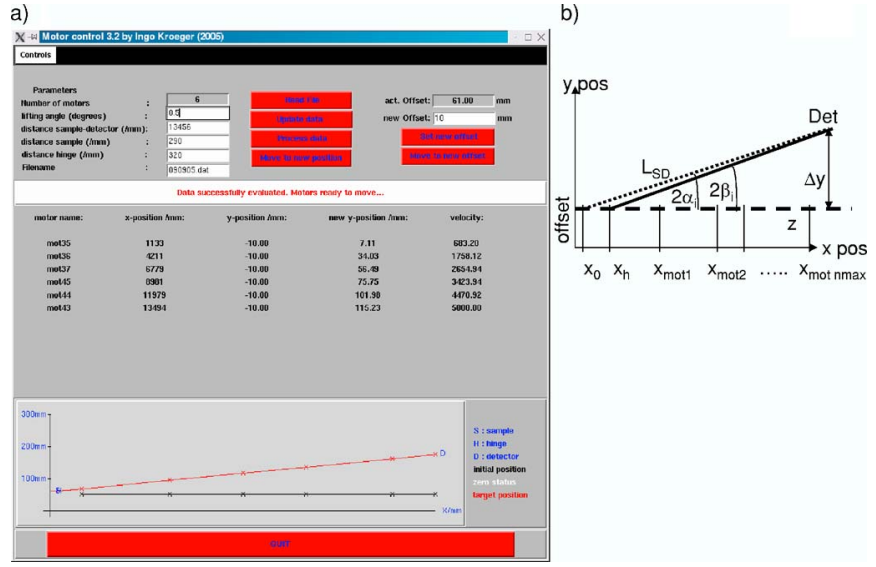

FIG. 2. (Color online) TK interface for tube movement in vertical direction. (a) Screen shot; (b) coordinate system.

The evacuated pipe which guides the radiation from the USAXS guard slit $S_{2}$ located at the entrance of the beam into the experimental hutch was reconstructed. It has been divided into six modules to take into account the various possible distances between the sample position and the detector. Each module is mounted on a height adjustable support driven by stepping motors. By this means the flight tube between sample and detector can either be lifted with respect to the incident beam to shift along the accessible $q$ range or it can be tilted to adapt to the inclined flight path in grazing incidence geometry. Positioning is easily controlled via a Perl script with TCL/TK-based graphical user interface (GUI) running under the HASYLAB standard experiment control software SPECTRA. ${ }^{32}$ A screen shot of the GUI is shown in Fig. 2(a). To attain a given inclination angle determined by the angle of reflection $\alpha_{i}$ or offset, all the necessary supports are moved simultaneously. The number and positions $x_{\text {mot, } j}$ $(j=1, \ldots, N<7)$ of the supports depend on the sample-todetector distance $L_{\mathrm{SD}}$ and are defined in a parameter file. The inclination $\beta_{i}$ is performed relative to a defined point of rotation, usually a hinge at variable position. Figure 2(b) shows the coordinate system used to rotate about the hinge. This motorized automation significantly reduces the time needed to switch from the SAXS setups in transmission geometry to GISAXS setups. Depending on detailed setup required, the tube movement is performed within minutes. The switch from transmission to reflection geometry including installation of GISAXS sample chamber and beam stop(s) and installation and alignment of the $\mu$-focus option (if required) can easily be accomplished within one shift. The flight tube consists of two parts having different diameters. The upstream part of $9.5 \mathrm{~m}$ length consists of DN160 ISO-K tubes, whereas the remaining $2.0 \mathrm{~m}$ close to the detector is formed by DN200 ISO-K tubes to obtain an increased aperture required by the (GI)USAXS geometry. ${ }^{9}$ A photograph of the reconstructed beamline is shown in Fig. 3. Using six tube modules allows the installation of the sample environment at five basic sample-to-detector distances between $1.8 \mathrm{~m}$ $\leqslant L_{\mathrm{SD}} \leqslant 12.7 \mathrm{~m}$. The length of the vertical traverse path of each support is $100 \mathrm{~mm}$.

Table I summarizes the various SAXS setups which can now be realized at BW4. It includes the different sample-to- 


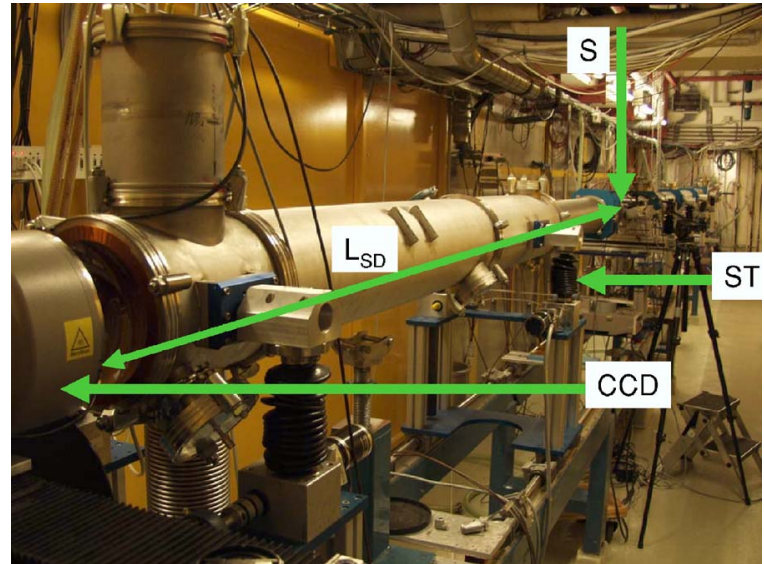

FIG. 3. (Color online) Photograph of BW4 as seen from detector position. One of the motorized supports is indicated by ST. CCD denotes the new CCD detector and $\mathrm{S}$ the sample position at $L_{\mathrm{SD}}=3.8 \mathrm{~m}$.

detector distances $L_{\mathrm{SD}}$, the corresponding minimum and maximum detectable length scales $\left(d_{\min }, d_{\max }\right)$, and primary beam size. The SAXS options are grouped into two categories, namely, microfocus beam and standard beam. The standard beam with $400 \times 400 \mu \mathrm{m}^{2}$ dimensions is produced by the slit collimation system together with the two focusing mirrors. The details of the microfocus option are described in Sec. IV.

When using a beam size of $400 \times 400 \mu \mathrm{m}^{2}$, the highest resolution is obtained at $L_{\mathrm{SD}}=12.4 \mathrm{~m}$, see Fig. 1. In transmission USAXS, the resolution is limited by the minimum size of the beam stop obscuring the direct beam, and the maximum detectable correlation distance is $d_{\max }=650 \mathrm{~nm}$ [Fig. 4(a)]. The primary beam stop is a rectangular shaped lead block with dimensions of $6 \mathrm{~mm}$ in the horizontal and $3 \mathrm{~mm}$ in the vertical direction. A photodiode in the center is used to monitor the primary beam intensity. The $d_{\max }$ values for transmission SAXS, USAXS, and $\mu$-SAXS given in Table I are achieved with the beam stop centered symmetrically relative to the primary beam profile. The values in parentheses are achieved in the vertical direction with an offcenter alignment, where the primary beam profile is close to one edge of the beam stop to observe the scattered intensity as close as possible to the primary beam. In GISAXS, GIUSAXS, and $\mu$-GISAXS, two separate pointlike beam stops are used to shield the specular reflected beam and the

TABLE I. Overview of the different SAXS configurations at BW4. The sample-detector distance is denoted by $L_{\mathrm{SD}}$, the minimum accessible structure size by $d_{\min }$, and the maximum accessible structure size by $d_{\max }$.

\begin{tabular}{lccll}
\hline \hline & $L_{\mathrm{SD}}(\mathrm{m})$ & $d_{\min }(\mathrm{nm})$ & $d_{\max }(\mathrm{nm})$ & Beam size $\left(\mu \mathrm{m}^{2}\right)$ \\
\hline SAXS & 1.8 & 3 & 90 & $400 \times 400$ \\
& 3.8 & 5 & 181 & $400 \times 400$ \\
GISAXS & 2.3 & 3 & $>300$ & $400 \times 400$ \\
USAXS & 8 & 10 & 450 & $400 \times 400$ \\
& 12.4 & 30 & $650(1 \mu \mathrm{m})^{\mathrm{a}}$ & $400 \times 400$ \\
GIUSAXS & 12.7 & 30 & $>7000$ & $400 \times 400$ \\
$\mu$-SAXS & 2.3 & 3 & $100(150)^{\mathrm{a}}$ & $65 \times 35$ \\
$\mu$-GISAXS & 2.3 & 3 & $>400$ & $65 \times 35$ \\
\hline
\end{tabular}

${ }^{\mathrm{a} B e a m}$ stop vertically off centered. a)

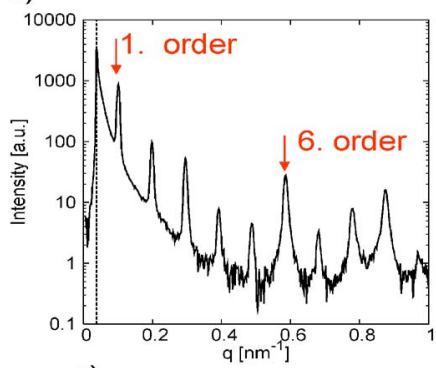

b)

c)
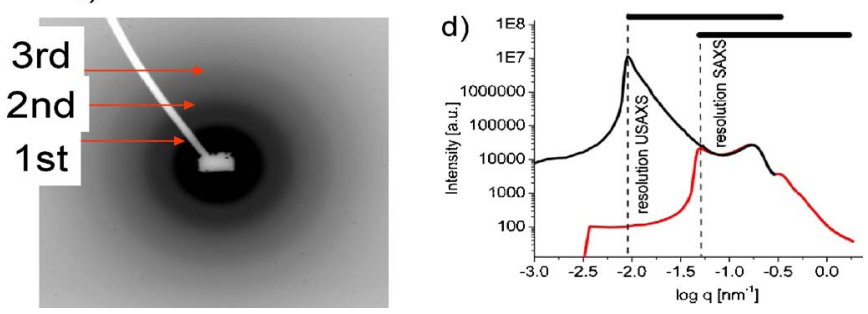

FIG. 4. (Color online) (a) Collagen pattern collected using a beam size of $B=65 \times 35 \mu \mathrm{m}^{2}$ produced by 10 beryllium compound refractive lenses (Be CRLs). The first order is clearly separated at $L_{\mathrm{SD}}=2.3 \mathrm{~m}$; the small-angle resolution is $d_{\max }=150 \mathrm{~nm}$. (b) 2D USAXS pattern of high-density polyethylene (HDPE, Lupolen by BASF) at $L_{\mathrm{SD}}=12.4 \mathrm{~m}$. (c) 2D SAXS pattern $\left(L_{\mathrm{SD}}=2.04 \mathrm{~m}\right)$ of Lupolen. Both patterns were recorded with the CCD detector. (d) Combined pie-shaped integrated USAXS and SAXS data in a double-logarithmic plot. The overlap region on the $q$ axis is large, and both curves can be perfectly combined. The accessible USAXS and SAXS regions are indicated by horizontal lines; the beam stop limits are denoted as resolution USAXS $\left(d_{\max }=650 \mathrm{~nm}\right)$ and resolution SAXS $\left(d_{\max }=125 \mathrm{~nm}\right)$.

primary beam which in this geometry does not affect the resolution as in the case of transmission geometry. ${ }^{20,21,24,25}$ Thus, in reflection setups, the relevant scattering information is not partially hidden behind a beam stop, and therefore the resolution is only limited by the divergence of the primary beam, the sample-to-detector distance $L_{\mathrm{SD}}$, and the spatial resolution of the detector. ${ }^{9,18}$

Each specific SAXS sample position listed in Table I requires a guard slit mounted close to the sample in order to optimize the achievable resolution by reducing parasitic scattering. ${ }^{26}$ For this purpose a new piezodriven portable slit system was constructed as shown in Fig. 5. It is housed in a vacuum compatible enclosure equipped with DN160 ISO-K flanges $\left(p<1 \times 10^{-6}\right.$ mbar possible) which can be attached to the flight tube modules at the various SAXS sample posi-

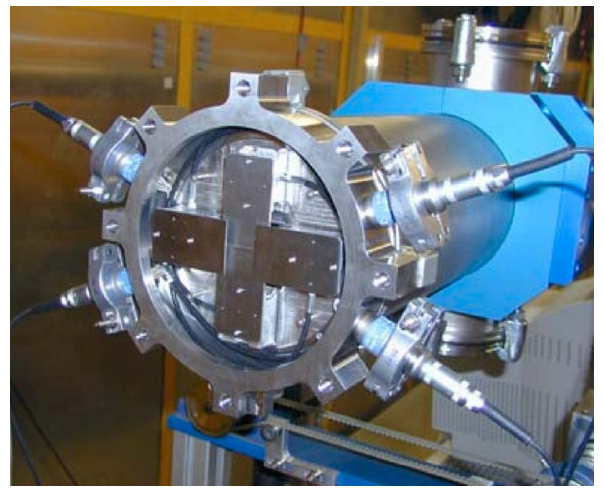

FIG. 5. Color online) Photograph of the piezodriven slit system used for the SAXS setup at BW4. 


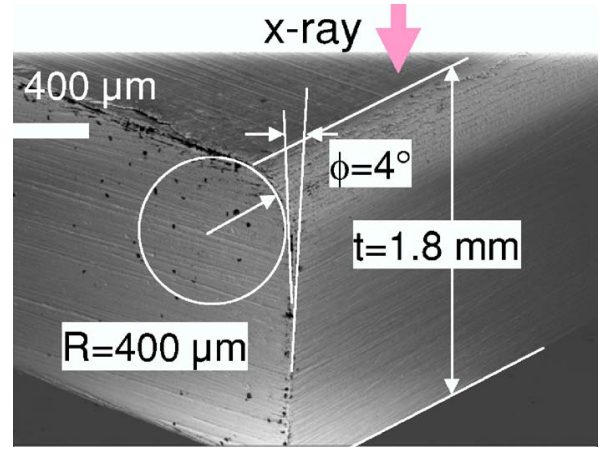

FIG. 6. (Color online) Scanning electron microscope (SEM) image of one individual blade of the piezo-slit-system. The geometry has been designed for optimum antiscattering and absorption.

tions listed in Table I. Each slit bracket is mounted on a piezodriven translation stage (travel range $30 \mathrm{~mm}$ each) with a nominal position resolution of $\Delta x= \pm 3 \mu \mathrm{m}$ and moves independently. The maximum achievable gap is $30 \times 30 \mathrm{~mm}^{2}$. Special care has been taken to design the blade edges. Figure 6 shows a scanning electron microscope (SEM) image of the blade surface. The design has been optimized for minimum background, maximum absorption, and easy production. It is manufactured from sintered tungsten powder $(99.95 \%$ purity, $2 \mathrm{~mm}$ thickness, W000370, Goodfellow GmbH). The major source of background is total reflection of the direct beam on the blade surface and scattering from the edges. ${ }^{33,34}$ To minimize these effects, the region of the blade touching the $\mathrm{x}$-ray beam has been chamfered by mechanical polishing to an entrance radius of $R=400 \mu \mathrm{m}$. Furthermore, to maximize absorption at the edges, the curved surface steadily passes over to a plain one which is inclined under an angle of $\phi=4^{\circ}$ with respect to the beam direction (Fig. 6).

Figures 4(b) and 4(c) show two scattering patterns obtained using a charge-coupled device (CCD) detector. The sample is high-density polyethylene [Lupolen by BASF (Ref. 30)] which serves as a calibration standard at BW4. The USAXS data were obtained at a sample-to-detector distance of $L_{\mathrm{SD}}=12.4 \mathrm{~m}$, while the SAXS data were taken with $L_{\mathrm{SD}}=2.04 \mathrm{~m}$. Three diffraction orders are clearly visible in the SAXS pattern. One-dimensional data obtained by pieshaped integration are shown in the plot combining both measurements. The data sets fit excellently to each other and exhibit a large range of overlapping $q$ values.

\section{DETECTOR AND BEAM MONITORING SYSTEM}

Besides the formerly used two-dimensional Gabriel-type gas detector, ${ }^{10,29}$ a commercial CCD detector [MARCCD 165 (Ref. 35)] went into operation at BW4 very successfully. The detector has an active area of $165 \mathrm{~mm}$ in diameter, which is reduced via glass fiber taper (2.7:1 demagnification ratio) to the CCD chip area having $2048 \times 2048$ pixels, each of $79.1 \mu \mathrm{m}$ pixel size. The detective quantum efficiency for $10 \mathrm{keV}$ photons is around $80 \%$ (as given by the manufacture $^{35}$ ). The nominal gain is about $6 e^{-}$/photon at the photon energy used, and the full well capacity is 4 $\times 10^{5} e^{-} /$pixel corresponding to $6 \times 10^{4}$ photons/pixel. The $\mathrm{CCD}$ chip is cooled down to $-70{ }^{\circ} \mathrm{C}$, thus featuring a very

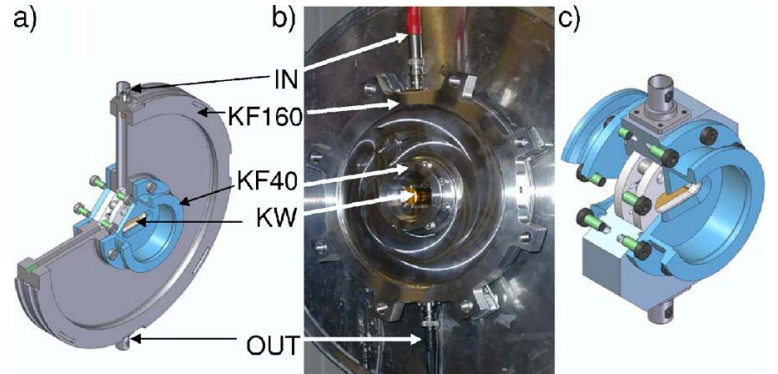

FIG. 7. (Color online) (a) Sketch of the new beam monitor. IN: high voltage input; KF160, KF40: flange adapters DN160 ISO-K and DN40 ISO-K; KW: Kapton window (thickness $t_{\mathrm{KW}}=150 \mu \mathrm{m}$ ); and OUT: signal output to amplifier. (b) Photograph of the installed device. (c) Sketch of the latest (small) KF40 version to reduce sample-to-monitor distance.

low dark current of less than $0.01 e^{-} /$pixel s and a readout noise of less than $10 e^{-} /$pixel at $3.5 \mathrm{~s}$ readout time. A mechanical fast shutter is installed in front of the sample to avoid irradiation of the detector during readout intervals. The minimum acquisition time is defined by the speed of the shutter and is about $100 \mathrm{~ms}$. Precise monitoring of the intensity of the incident and transmitted direct beams is essential for data evaluation in small-angle scattering experiments. ${ }^{5}$ Ionization chambers are well suited for this purpose. Figure 7(a) shows the design of the actual standard multiflange ionization chamber with kapton windows (thickness: $25 \mu \mathrm{m}$ ). The width of the windows is $25 \mathrm{~mm}$, the height is $15 \mathrm{~mm}$, the length of the air path is $20 \mathrm{~mm}$. Figure 7(b) shows a photograph of this routinely used ionization chamber. It can be mounted at the end of an adequate flight tube segment for intensity monitoring behind all optical elements. The monitor can be attached directly to KF160 or KF40 flanges, respectively, due to its multiflange adapters. Alternatively, the design features four holes in the body between the active monitor area and the flange area which allow to connect the vacuum between the adjacent segments on both sides of the monitor. A version of these monitors is especially designed for situations where the sample environment is not directly connected to the flight tube vacuum and the monitor acts as an exit or entrance window. It is shown in Fig. 7(c). This chamber is installed in a housing with KF40 flanges on both sides. Due to its reduced space requirements, it allows to realize a small sample-to-monitor distance which is mainly limited by the sample environment only.

\section{MICROFOCUS OPTION}

With the standard optics of BW4, the typical beam size is $400 \times 400 \mu \mathrm{m}^{2}{ }^{10}$ The CCD detector has a five times smaller spatial resolution than the formerly used wire detector. Thus the CCD is able to make use of the increase of SAXS resolution capabilities if the primary beam cross section is reduced at the detector. On the other hand, for many applications, a reduction of the beam size at the sample position is desirable. The high resolution, high flux, and excellent beam stability offered by the BW4 beamline allow to introduce microfocusing optics such as compound refractive lenses. In a pilot experiment we have chosen parabolic compound refractive lenses made of beryllium ${ }^{36}$ (Be CRLs) to 


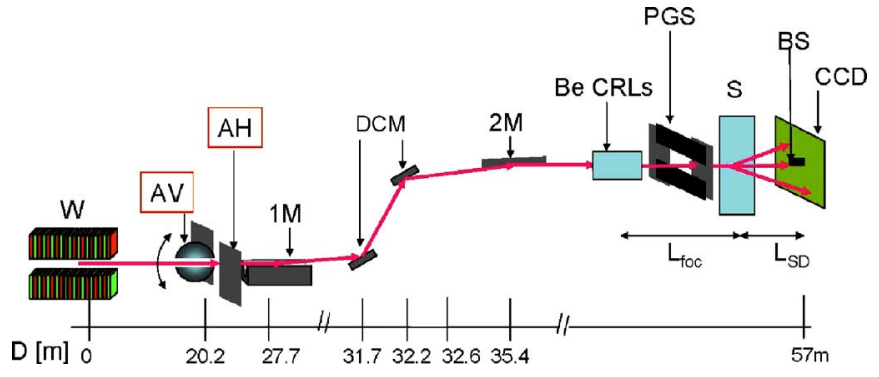

FIG. 8. (Color online) Sketch of the BW4 $\mu$-focus setup including beryllium compound refractive lenses (Be CRLs). D: distance to wiggler; W: wiggler; $\mathrm{AV}, \mathrm{AH}$ : adjustable aperture in vertical and horizontal direction; 1M: horizontally focusing mirror; DCM: Si(111) double crystal monochromator; 2M: planar mirror; Be CRLs: beryllium compound refractive lenses; PGS: peizodriven guard slit system; S: sample position; BS: beam stop for direct beam; CCD: MARCCD detector; $L_{\text {foc }}$ : focal length of Be CRLs $(2.5 \mathrm{~m})(1.7 \mathrm{~m})$ for $N=10(15) \mathrm{BeCRLs}$; and $L_{\mathrm{SD}}$ : sample-detector distance.

reduce the beam spot size at the sample position. There exist many other options to produce micrometer-sized spots, e.g., Kirkpatrick-Baez-mirrors (KBM) ${ }^{37}$ Fresnel zone plates (FZPs), ${ }^{38}$ and pinholes. We have chosen Be CRLs as being well suited for the wiggler beamline due to the following reasons. First of all, a simple pinhole collimation using pinhole sizes in the $10 \mu \mathrm{m}$ range is not reasonable due to the tremendous geometric losses in flux inflicted by such a setup. Fresnel zone plates,$^{38}$ having slightly smaller acceptance compared to Be CRLs, require a more complicated alignment. Be CRLs are on-axis optics. Thus the beam path is not angularly offset, neither vertically nor horizontally, and the geometry of the beamline is not altered. Moreover, the focal spot size can be adapted to the experiment by changing the number of lenses. However, Be CRLs such as FZPs are achromatic optical devices. A change in energy thus requires a change in number of and position of the Be CRLs. Both can be accomplished in short time due the flexibility of the lens holder and the flight path setup. Vibrations of the lenses itself or source movements are reduced by the demagnification ratio $m$ when using Be CRLs, in contrast to mirrors, where such movements are directly transmitted to a movement of the focal spot due to the deflection geometry.

The lenses have a radius of curvature $R=0.2 \mathrm{~mm}$ at the apex of the paraboloid and a geometric aperture $a$ $=0.95 \mathrm{~mm}$. Beryllium allows for high transmission $(>50 \%)$ at the standard wavelength of $\lambda=0.138 \mathrm{~nm}$. At present, 10 or 15 CRLs can be routinely installed on demand at a position $L_{1}=30 \mathrm{~m}$ or $L_{1}=32 \mathrm{~m}$ behind the first beam defining optical element, an absorber acting simultaneously as an adjustable aperture. It defines the initial beam size which is imaged on the sample in a microfocus setup, see Fig. 8. The focal distance of the setup is $L_{\mathrm{foc}}=R /(2 N \delta)$ $=2.5 \mathrm{~m}$ for $N=10$ and $L_{\text {foc }}=1.7 \mathrm{~m}$ for $N=15$ Be CRLs, with $\delta$ being the real part of the refractive index for $8.9 \mathrm{keV}$ and $N$ being the number of lenses. The demagnification ratio is then $m \approx 10$.

With $N=10$ Be CRLs, the beam spot dimensions at the sample result in $65 \mu \mathrm{m}$ in the horizontal and $35 \mu \mathrm{m}$ in the vertical direction with an absorber opening of 0.4 $\times 0.33 \mathrm{~mm}^{2}$. The resulting beam sizes can be calculated according to
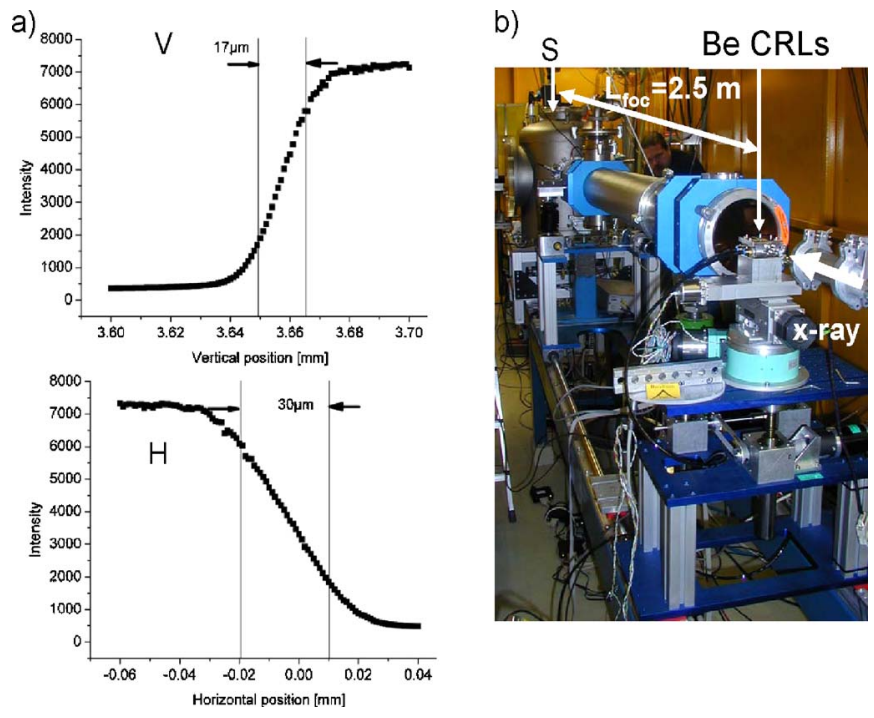

FIG. 9. (Color online) (a) Knife-edge scans for determining beam sizes of the $\mu$-focus option. The minimum beam size obtained at BW4 is $B=30$ $\times 17 \mu \mathrm{m}^{2}$ using $15 \mathrm{Be}$ CRLs. (b) Photograph of BW4 with the $\mu$-focus setup. The position of the Be CRLs is marked by an arrow. Except for the Be CRLs, all flight paths are evacuated.

$$
G / g=B / L_{2},
$$

with $G$ being the size of the source imaged, $g$ its distance to the Be CRLs, $L_{2}$ the distance of the image to the Be CRLs, and $B$ the resulting beam spot size in the focus. $L_{2}$ can be calculated according to

$$
1 / L_{1}+1 / L_{2}=1 / L_{\text {foc }}
$$

In the horizontal direction the beam size is twice as large as one would estimate for a setup without a horizontally focusing mirror. For this experiment the two mirrors of BW4 stayed in the beam, the second one set to a planar nonfocusing geometry while the first one still performed horizontal focusing. Thus the Be CRLs image in the horizontal direction the virtual source produced by the mirror. Removing both mirrors is foreseen for future experiments, reducing the horizontal beam size by a factor of 2 , but this option will lead to a different entrance height of the beam into the experimental hutch which requires additional changes of the setup (e.g., a new entrance window).

Figure 4(a) shows a collagen $\mu$-SAXS pattern obtained with the $65 \times 35 \mu \mathrm{m}^{2}$ beam. No additional collimators were used. The first order reflection of the $65 \mathrm{~nm}$ periodic structure is clearly separated from the beam stop; the resolution limit is $d_{\max }=150 \mathrm{~nm}$ at the given sample-to-detector distance of $L_{\mathrm{SD}}=2.337 \mathrm{~m}$. The guard slits described above were only used to reduce parasitic scattering. In order to test whether the beam size can be further reduced without additional collimation, a total number of $N=15$ Be CRLs was also used. In this case a minimum beam size of 30 $\times 17 \mu \mathrm{m}^{2}$ can be reached as shown in Fig. 9(a). However, the absorption of the Be CRLs is already significant for this setup (transmission $<25 \%$ ). 


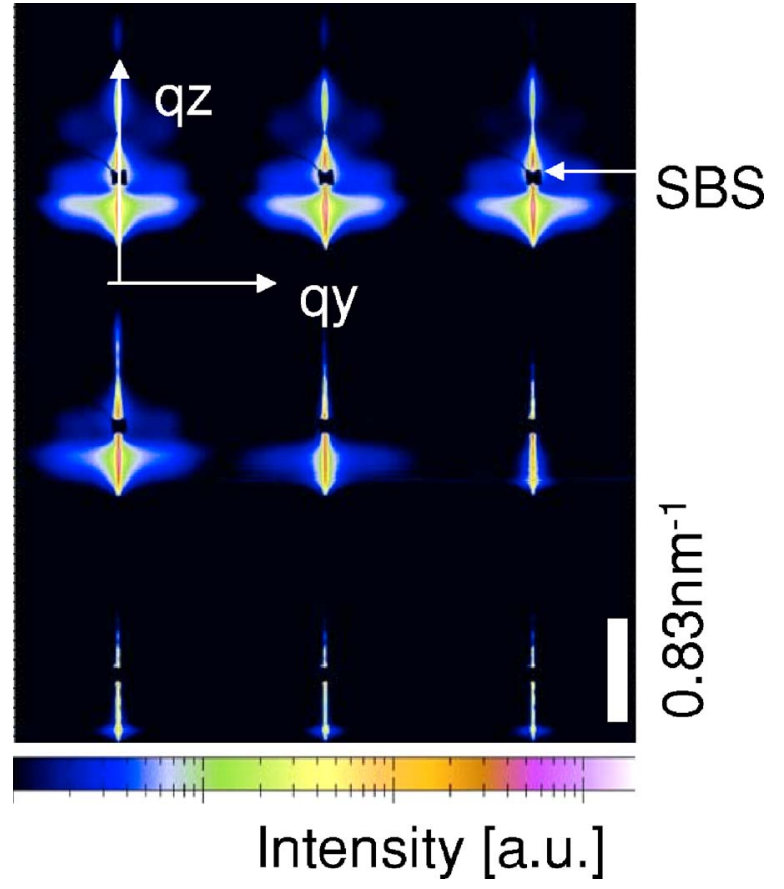

FIG. 10. (Color online) Micro grazing incidence small-angle x-ray scattering pattern of a standard gradient gold $(\mathrm{Au}) /$ polystyrene/silicon $(\mathrm{Si})$ multilayer. The step width of the scanning is $\Delta y=70 \mu \mathrm{m}$; the beam size $B$ $=65 \times 35 \mu \mathrm{m}^{2}$. The specular beam stop (SBS) is indicated. The direct beam stop is not visible in the $\mu$-GISAXS pattern. Nine $2 \mathrm{D}$ scattering pattern are grouped in the $3 \times 3$ matrix by starting in the fully gold nanoparticle covered part of the gradient in the upper left and ending in the uncovered PS part of the gradient in the lower right corner.

\section{APPLICATION EXAMPLE: $\mu$-GISAXS}

$\mu$-SAXS is a commonly used technique to investigate hierarchical structures on multiple length scales. ${ }^{39-42}$ Recently $\mu$-SAXS was combined with a reflection setup resulting in a new method called microbeam GISAXS $\left(\mu\right.$-GISAXS). ${ }^{21,43,44}$ As an initial application for the moderately focused microbeam at BW4, we carried out $\mu$-GISAXS experiments at this instrument. Focusing on the sample position allows to reduce the sample size to $1 \mathrm{~mm}$, i.e., a decrease in size by one order of magnitude. At the same time the signal-to-noise ratio is increased. A simple vertical beam size reduction using slits would have led to significant intensity losses. Furthermore, by using a microbeam, combinatorial mapping becomes possible.

To establish and prove the feasibility of $\mu$-GISAXS, we applied this technique to a gradient gold $(\mathrm{Au}) /$ polystyrene (PS) double layer on top of a silicon $(\mathrm{Si})$ substrate. ${ }^{21} \mathrm{We}$ established a fully evacuated setup to avoid air scattering, see Fig. 9(b). Only the air path remained on both sides of the Be CRLs, as the lens holder compartment is presently still operated under inert gas. In the case of a moderate microfocus, as it is available in our setup, only one-dimensional (1D) gradients in the direction perpendicular to the beam are easily accessible. It should be noted that the beam focused by the Be CRLs is no further collimated.

Figure 10 shows nine two-dimensional (2D) $\mu$-GISAXS patterns obtained by scanning along the 1D gradient multilayer (starting in the gold covered part). They are represented on a logarithmic intensity scale as a function of two
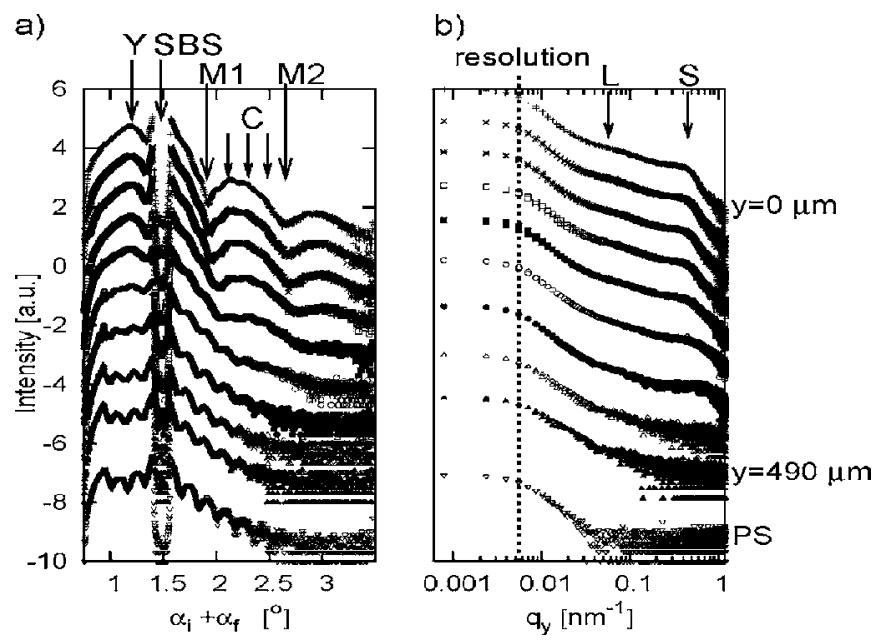

FIG. 11. Line scans cut from the 2D pattern. (a) Detector scans. SBS denotes the specular peak being shadowed by a pointlike lead beam stop. C denotes the correlated roughness fringes and M1 and M2 the fringes due to the height of the Au nanoparticles. Y denotes the Yoneda peak of gold. (b) Out-of-plane scans. L and S denote positions of the structure factor maxima of the large and small nanoparticles. In both pictures, the curves are shifted for clarity. PS represent the scattering pattern from the pure PS surface. The beginning of the gradient is situated at $y=0 \mu \mathrm{m}$ in the completely Au covered region.

perpendicular projections of the momentum transfer, namely, $q_{z}=(2 \pi / \lambda) \sin \left(\alpha_{i}+\alpha_{f}\right)$ and $q_{y}=(2 \pi / \lambda) \sin \Psi \cos \left(\alpha_{f}\right)$, where $\Psi$ denotes the out-of-plane angle parallel to the sample surface, $\alpha_{f}$ the exit angle vertical to the sample surface, and $\alpha_{i}$ the incident angle of the primary beam. Corresponding to the horizontal beam dimension, the step width was $\Delta y=70 \mu \mathrm{m}$. Along the gradient, the $\mu$-GISAXS signal clearly changes due to the decreasing mass thickness of the gold layer. The first 2D scattering patterns are dominated by the strong scattering from the gold nanoparticles. With reducing amount of gold, along the gradient, the scattering features change. Without gold on the surface, at the end of the scan, the typical 2D scattering pattern of a homogeneous PS film on $\mathrm{Si}$ remains. In all 2D data, the specular beam stop is clearly visible as a square shaped shadow. Instead of a model fitting of the full 2D intensity maps, ${ }^{21}$ which is beyond the scope of this article, we restrict the analysis to selected cuts out of the 2D intensity. ${ }^{18}$

Figure 11(a) shows the detector scans (vertical cuts) on a semilogarithmic scale as a function of the detector angle $\alpha_{i}$ $+\alpha_{f}$. The incident angle $\alpha_{i}$ was fixed to $\alpha_{i}=0.75^{\circ}$. The detector scans show two characteristic features, related to electron density fluctuation perpendicular to the sample surface (parallel to $q_{z}$ ) indicated by arrows. Firstly, the form factor of the nanoparticles along $q_{z}$, i.e., the height of the nanoparticles, manifests itself as large fringes (corresponding minima are denoted with M1 and M2). Thus the nanoparticle height can be deduced from the fringes seen in Fig. 11(a). It depends, however, on the detailed shape of the nanoparticles. ${ }^{21}$ It is obvious that the height of the nanoparticles decreases along the gradient. Secondly the correlated roughness originated from the PS layer is evidently visible as small fringes (corresponding minima are denoted with $\mathrm{C}$ ). This so-called resonant diffuse scattering (RDS) arises from partial coherent scattering of the waves at different interfaces 
(Au/PS and PS/Si). In the present multilayer system, at $\lambda$ $=0.138 \mathrm{~nm}$, PS has the lowest index of refraction. The interference maxima fulfill the one-dimensional Bragg condition $\Delta q_{z}=2 \pi / d_{\text {corr }}$. This leads to $d_{\text {corr }}=47 \mathrm{~nm}$ in our case, which corresponds well to a nominal thickness of $40 \mathrm{~nm}^{21}$ With increasing gold layer thickness (bottom to top), the RDS fringes of the PS layer are reduced because the scattering of the gold nanoparticles is becoming dominant. For $\alpha_{f}<\alpha_{i}$ the RDS is completely suppressed by the strong Yoneda peak from gold (denoted with Y). This intensity peak occurs when $\alpha_{i}$ or $\alpha_{f}$ equal the critical angle $\alpha_{c}$ of the material. $\alpha_{c}$ can be calculated via the real part $\delta$ of the complex index of refraction $n=1-\delta+i \beta$ by $\alpha_{c}=(2 \delta)^{0.5}$. Thus the critical angle is energy dependent, and for gold $\alpha_{c}=0.49^{\circ}$ at $0.138 \mathrm{~nm}$ wavelength.

Figure 11(b) shows the out-of-plane scans cut from the 2D intensity. As described in Refs. 21 and 45, a bimodal distribution of nanoparticle classes is present: large nanoparticles (diameter $2 R_{L}>30 \mathrm{~nm}$, distance $D_{L}>110 \mathrm{~nm}$ ) and small, closely packed ones $\left(2 R_{S} \approx 10 \mathrm{~nm}, D_{S}=14 \mathrm{~nm}\right)$. The corresponding interference maxima are marked by arrows. Their position stays constant throughout the gradient. The diffuse scattering stemming from the interference of large and small clusters $\left(D_{L S}=R_{L}+R_{S}\right)$ leads to a broad intensity distribution between the two arrows, since the size distribution is nonuniform. Thus strong changes in the intensity arise, visible in Fig. 12 (b), when scanning along the gradient.

\section{OUTLOOK}

The instrumentation at beamline BW4 has undergone significant refurbishment and upgrading. In addition to the standard USAXS geometry, additional SAXS setups are possible, thus increasing the accessible $q$ range. Major effort has been made to design and construct a modular and flexible collimation system for the shorter sample-to-detector distances, such that intensity and resolution can be optimized for each individual setup. The accessible $q$ range corresponds to real space length scales $3 \mathrm{~nm} \leqslant d \leqslant 1 \mu \mathrm{m}$. Exploiting the high flux and the low divergence of BW4, microfocusing optics can be utilized. The new portable low-background guard slits with $\pm 3 \mu \mathrm{m}$ positioning resolution are perfectly adapted to the small beam size. The option of a moderate microfocus for $\mu$-SAXS experiments allows performing onedimensional combinatorial investigations.

\section{ACKNOWLEDGMENTS}

The authors thank A. Fröhmsdorf and A. Kornowski for preparing the SEM image.

${ }^{1}$ O. Glatter and O. Kratky, Small Angle X-ray Scattering (Academic, New York, 1982).

${ }^{2}$ L. A. Feigin and D. I. Svergun, Structure Analysis by Small-Angle X-ray and Neutron Scattering (Plenum, New York, 1987).

${ }^{3}$ J. S. Pedersen, Adv. Colloid Interface Sci. 70, 171 (1997).

${ }^{4}$ P. Fratzl, J. Appl. Crystallogr. 36, 397 (2003).

${ }^{5}$ Neutrons, X-rays and Light: Scattering Methods Applied to Soft Condensed Matter, edited by P. Lindner and Th. Zemb (North-Holland, Am- sterdam, 2002).

${ }^{6}$ H. G. Haubold, in Streumethoden zur Untersuchung Kondensierter Materie, edited by R. Hölzle (Forschungszentrum Jülich GmbH, Jülich, 1996), pp. C6.1-C6.30.

${ }^{7}$ B. Chu and B. S. Hsiao, Chem. Rev. (Washington, D.C.) 101, 1727 (2001).

${ }^{8}$ P. Müller-Buschbaum, J. Phys.: Condens. Matter 15, R1549 (2003).

${ }^{9}$ P. Müller-Buschbaum, Anal. Bioanal. Chem. 376, 3 (2003).

${ }^{10}$ Z. Wang, B. S. Hsiao, N. Stribeck, and R. Gehrke, Macromolecules 35, 2200 (2002)

${ }^{11}$ U. Lode, T. Pomper, A. Karl, G. v. Krosigk, S. Cunis, W. Wilke, and R. Gehrke, Macromol. Rapid Commun. 19, 35 (1998).

${ }^{12}$ M. Rössle, D. Flot, J. Engel, M. Burghammer, C. Riekel, and H. Chanzy, Biomacromolecules 4, 981 (2003).

${ }^{13}$ G. Beaucage, H. K. Kammler, R. Mueller, R. Strobel, N. Agashe, S. E. Pratsinis, and T. Narayanan, Nat. Mater. 3, 370 (2004).

${ }^{14}$ F. J. Torre, M. M. Cortázar, M. A. Gómez, G. Ellis, and C. Marco, Polymer 44, 5209 (2003).

${ }^{15}$ A. Almendarez Camarillo, S. V. Roth, P. Bösecke, S. Buchner, K. Krenn, R. Gehrke, and N. Stribeck, Macromolecules (submitted)

${ }^{16}$ N. Stribeck, P. Bösecke, R. Bayer, and A. Almendarez Camarillo, Prog. Colloid Polym. Sci. 130, 127 (2005).

${ }^{17}$ E. Maurer, S. Loi, D. Wulff, N. Willenbacher, and P. Müller-Buschbaum, Physica B 357, 144 (2005).

${ }^{18}$ P. Müller-Buschbaum et al., Europhys. Lett. 42, 517 (1998),

${ }^{19}$ P. Müller-Buschbaum and M. Stamm, Macromolecules 31, 3686 (1998).

${ }^{20}$ P. Müller-Buschbaum, J. S. Gutmann, C. Lorenz-Haas, O. Wunnicke, M. Stamm, and W. Petry, Macromolecules 35, 2017 (2002).

${ }^{21}$ S. V. Roth, M. Burghammer, C. Riekel, P. Müller-Buschbaum, A. Diethert, and P. Panagiotou, Appl. Phys. Lett. 82, 1935 (2003).

${ }^{22}$ V. Holy and T. Baumbach, Phys. Rev. B 49, 10668 (1994).

${ }^{23}$ T. Salditt, T. H. Metzger, J. Peisl, and G. Goerigk, J. Phys. D 28, A236 (1995).

${ }^{24}$ P. Müller-Buschbaum, J. S. Gutmann, M. Stamm, R. Cubitt, S. Cunis, G. v. Krosigk, R. Gehrke, and W. Petry, Physica B 283, 53 (2000).

${ }^{25}$ P. Müller-Buschbaum, J. S. Gutmann, and M. Stamm, Phys. Chem. Chem. Phys. 1, 3857 (1999).

${ }^{26}$ G. Elsner, C. Riekel, and H. G. Zachmann, Adv. Polym. Sci. 67, 3 (1985).

${ }^{27}$ M. Müller, M. Burghammer, and C. Riekel, Nucl. Instrum. Methods Phys. Res. A 467-468, 958 (2001).

${ }^{28}$ R. Gehrke, Rev. Sci. Instrum. 63, 455 (1992).

${ }^{29}$ R. Gehrke, M. Bark, D. Lewin, and S. Cunis, Rev. Sci. Instrum. 66, 1354 (1995).

${ }^{30}$ A. Endres, U. Lode, G. v. Krosigk, M. Bark, S. Cunis, R. Gehrke, and W. Wilke, Rev. Sci. Instrum. 68, 4009 (1997).

${ }^{31} \mathrm{http}: / /$ www-hasylab.desy.de/facility/experimental_stations/BW4/BW4.htm

${ }^{32}$ I. Kröger, Project work, summer student program, 20/07/05-13/09/05, 2005 (unpublished).

${ }^{33}$ E. Vlieg, S. A. De Vries, J. Alvarez, and S. Ferrer, J. Synchrotron Radiat. 4, 210 (1997)

${ }^{34}$ D. Le Bolloc'h, F. Livet, F. Bley, T. Schulli, M. Veron, and T. H. Metzger, J. Synchrotron Radiat. 9, 258 (2002).

${ }^{35} \mathrm{http}: / / \mathrm{www} . \mathrm{marresearch.com} /$

${ }^{36}$ B. Lengeler et al., J. Phys. D 38, A218 (2005).

${ }^{37}$ O. Hignette et al., Proc. SPIE 4499, 105 (2001)

${ }^{38}$ C. David, T. Weitkamp, B. Nöhammer, and J. F. van der Veen, Spectrochim. Acta, Part B 59, 1505 (2004).

${ }^{39}$ S. V. Roth, M. Burghammer, A. Diethert, and P. Müller-Buschbaum, J. Appl. Crystallogr. 36, 684 (2003).

${ }^{40}$ M. Müller, C. Czihak, M. Burghammer, and C. Riekel, J. Appl. Crystallogr. 33, 817 (2000).

${ }^{41}$ O. Paris, D. Loidl, H. Peterlik, M. Müller, H. Lichtenegger, and P. Fratzl, J. Appl. Crystallogr. 33, 695 (2000).

${ }^{42}$ C. Lorenz-Haas, P. Müller-Buschbaum, O. Wunnicke, C. Cassignal, M. Burghammer, C. Riekel, and M. Stamm, Langmuir 19, 3056 (2003).

${ }^{43}$ P. Müller-Buschbaum, S. V. Roth, M. Burghammer, A. Diethert, P. Panagiotou, and C. Riekel, Europhys. Lett. 61, 639 (2003).

${ }^{44}$ S. V. Roth et al., Appl. Phys. Lett. 88, 021910 (2006).

${ }^{45}$ S. V. Roth, P. Müller-Buschbaum, M. Burghammer, H. Walter, P. Panagiotou, A. Diethert, and C. Riekel, Spectrochim. Acta, Part B 59, 1765 (2004). 